\title{
Three ultrasound phenotypes of non-invasive follicular thyroid neoplasm with papillary-like nuclear features proposed for imaging-pathology analysis: single center experience
}

\author{
Ruifeng Liu ${ }^{1}$, Luying Gao ${ }^{1}$, Yu Xia ${ }^{1}$, Xiaoyi Li $^{2}$, Quan Liao ${ }^{2}$, Ying Jiang ${ }^{3}$, Qing Zhang ${ }^{1}$, Cheng Chen ${ }^{1}$, \\ Li Zhang ${ }^{1}$, Zhiyong Liang ${ }^{3}$, Yuxin Jiang ${ }^{1}$
}

${ }^{1}$ Department of Ultrasound, Peking Union Medical College Hospital, Chinese Academy of Medical Sciences and Peking Union Medical College, Beijing, China; ${ }^{2}$ Department of General Surgery, Peking Union Medical College Hospital, Chinese Academy of Medical Sciences and Peking Union Medical College, Beijing, China; ${ }^{3}$ Department of Pathology, Peking Union Medical College Hospital, Chinese Academy of Medical Sciences and Peking Union Medical College, Beijing, China

Contributions: (I) Conception and design: Y Xia, R Liu, X Li, L Gao; (II) Administrative support: Y Jiang, Y Xia; (III) Provision of study materials or patients: X Li, Q Zhang, Q Liao, Z Liang, Y Jiang; (IV) Collection and assembly of data: R Liu, C Chen, L Zhang; (V) Data analysis and interpretation: R Liu; (VI) Manuscript writing: All authors; (VII) Final approval of manuscript: All authors.

Correspondence to: Yu Xia; Yuxin Jiang. Ultrasound department, Peking Union Medical College Hospital, Chinese Academy of Medical Sciences and Peking Union Medical College, Shuaifuyuan 1\#, Dongcheng District, Beijing 100730, China. Email: xiayupumch@126.com; jiangyuxinxh@163.com.

Background: The terminology "non-invasive follicular thyroid neoplasm with papillary-like nuclear features" (NIFTP) was introduced to reduce overtreatment of thyroid carcinomas with indolent behavior. However, limited literature discussed the sonographic features of the entity in depth. The objective of this study is to summarize sonographic subtypes of NIFTP for precise diagnosis.

Methods: From January 2017 to June 2020, 13,531 consecutive patients underwent surgery for thyroid nodules were reviewed; 30 patients $(0.22 \%)$ with 30 NIFTP were eligible for this retrospective observational study. We evaluated ultrasound features of all the lesions and distributed them into 3 major types of ultrasound appearance using pattern recognition. Systemic literature review concerning ultrasonography of NIFTP was also performed.

Results: At sonography, all the NIFTP lesions were classified into three types: 19 (63.3\%) were categorized into type A-oval and solid nodule without other high-suspicion features, 7 (23.3\%) into type B-partially cystic mass without high-suspicion features, 4 (13.3\%) into type C-hypoechoic solid nodule with highsuspicion features including irregular margins, microcalcifications, taller-than-wide shape.

Conclusions: Three ultrasound patterns were proposed by us for ultrasound-cytopathology correlation analysis.

Keywords: Non-invasive follicular thyroid neoplasm with papillary-like nuclear features (NIFTP);
ultrasonography; 2015 American Thyroid Association management guideline; thyroid imaging reporting and data
system

Submitted Jul 16, 2020. Accepted for publication Nov 18, 2020.

doi: $10.21037 /$ gs-20-612

View this article at: http://dx.doi.org/10.21037/gs-20-612

\section{Introduction}

Nikiforov et al. in 2016 firstly termed NIFTP to reduce overtreatment of a group of highly indolent thyroid "cancer", non-invasive encapsulated follicular variant of papillary thyroid carcinoma (EFVPTC) (1). The tumor was previously classified in the follicular variant of PTC, and it harbors a more favored prognosis with scarce recurrence, metastases and death from disease compared with its 
counterparts-infiltrative FVPTC and invasive encapsulated FVPTC (2). According to the modified diagnostic criteria, clear demarcation with lack of invasion is the prerequisite of NIFTP, while the follicular growth pattern, nuclear features scoring of 2 or 3 , no necrosis and no high mitotic activity jointly determine its nature as a borderline neoplasm (3). In the 2017 World Health Organization (WHO) Classification of Tumors of Endocrine organ book (4), NIFTP was categorized into a section entitled 'Other encapsulated follicular patterned thyroid tumors', suggesting its acceptance among specialists. Although NIFTP gets rid of the suffix of 'carcinoma', it still holds very low malignant potential as the lymph node metastases and lung metastases of NIFTP were identified in previous studies $(5,6)$.

Preoperative ultrasound and subsequent US-guided fine needle aspiration are the mainstay procedures for evaluation of suspected thyroid nodule. We hold the opinion that the accurate recognition of NIFTP preoperatively should not only depend on cytopathological results by biopsy, but also referring to ultrasound findings for comprehensive analysis. As for cytopathological results, Bethesda categories 3 [atypia of undetermined significance/follicular lesion of undetermined significance (AUS/FLUS)], 4 [follicular neoplasm/suspicious for follicular neoplasm (FN/SFN)] and 5 (suspicious for malignancy) are the most frequent findings of NIFTP (7-10). In terms of US presentation, NIFTP always shows less risk features than classical PTC and radiologists may prone to divide NIFTP into benign groups in previous researches (11-13). Thus, it seems either methods have limited power in the assessment of NIFTP. However, few articles drilled the US findings of NIFTP particularly and in depth. As the first-line modality, more understanding of the nodule such as detailed US appearance subtypes would shed light of some confusion from equivocal cytopathological indications and facilitate preoperative diagnosis combined with clinical features. Therefore, we summarized US features of NIFTP on the basis of our data as well as published literature, aiming to unravel the US subtypes of the entity for radiology-histology correlation analysis. We present the following article in accordance with the STROBE reporting checklist (available at http:// dx.doi.org/10.21037/gs-20-612).

\section{Methods}

\section{Patients}

This study was conducted in accordance with the
Declaration of Helsinki (as revised in 2013). The study was approved by the Ethics Committee of Peking Union Medical College Hospital (No. S-K1304) and individual consent for this retrospective analysis was waived. Between January 2017 to June 2020, 41 patients with 41 NIFTP were identified among 13,531 all histological variants of papillary thyroid carcinoma $(41 / 13,531,0.30 \%)$. Of the 41 cases, 11 patients were excluded from the study for the absence of matched US-surgery-pathology data- 8 did not have US records of the lesions, 3 did not receive operation in our center. We finally included 30 patients with 30 NIFTP into the retrospective analysis (Figure 1). Their medical records and imaging data were reviewed in a continuously maintained database. The histologic criteria for the diagnosis of NIFTP were as following: (I) welldemarcated tumor without any suspected sign of capsular or vascular invasion; (II) follicular growth pattern and nuclear features of papillary thyroid carcinoma; (III) none papillae or psammoma bodies, necrosis and less than three mitoses per 10 high-power fields (400x) (Figure 2). All the patients enrolled must meet the prerequisites below: (I) preoperative US localization and characterization of every thyroid nodule; (II) undergone surgical procedure with operation documents; (III) affirmative pathology results in our center. Patients ill with multiple thyroid lesions should get each nodule exactly screened prior to surgery. Meanwhile, the operation findings of every nodule must in line with the US and pathology discovery.

\section{Ultrasound examination}

Most of the patients prior to surgery for thyroid nodule in our center had undergone ultrasound examination and the US data were stored in an imaging archiving system. After retrospectively screening, all the patients diagnosed as NIFTP by histopathology and equipped with US imaging were selected. Philips iU22 5-12 MHz or GE Logiq 9 6-15 MHz linear probe were used for examination for patients in supine position. A resident collected information from the original ultrasound reports on pre-defined US characteristics and retrieved images for analysis. Two radiologists specialized in thyroid field with 8 and 20 years of experience reappraised these nodules to identify variable features not described in the original report. All the NIFTP lesions were sorted into two groups by size measured at sonography, with lesion size $\geq 1$ and $<1 \mathrm{~cm}$ in macrogroup and micro-group, respectively. US features included localization, size, echogenicity, composition, shape, margin, 


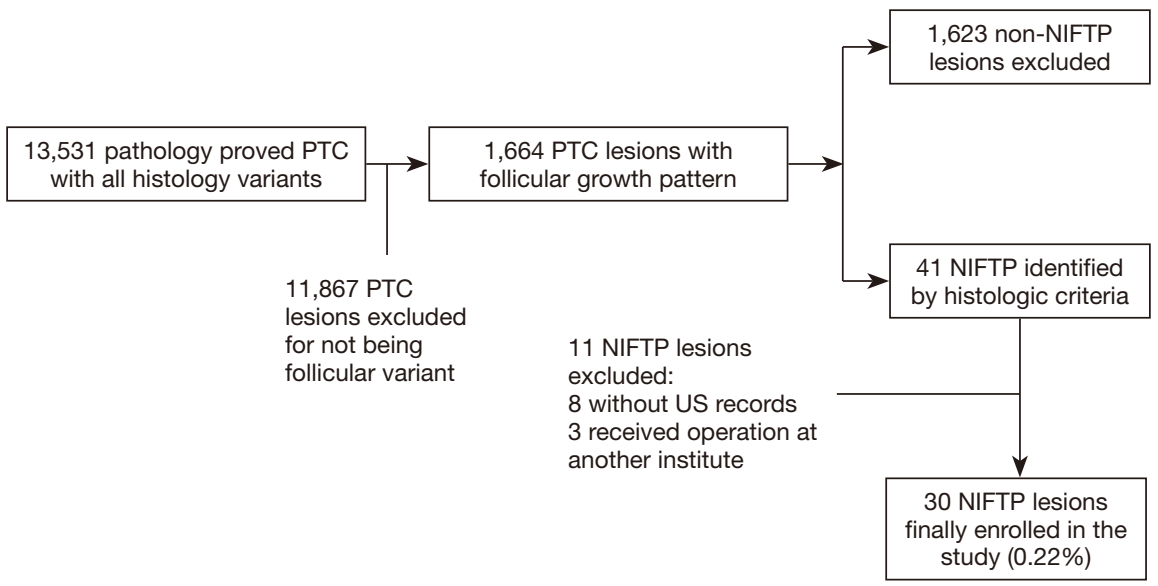

Figure 1 Flow scheme of patients' selection.
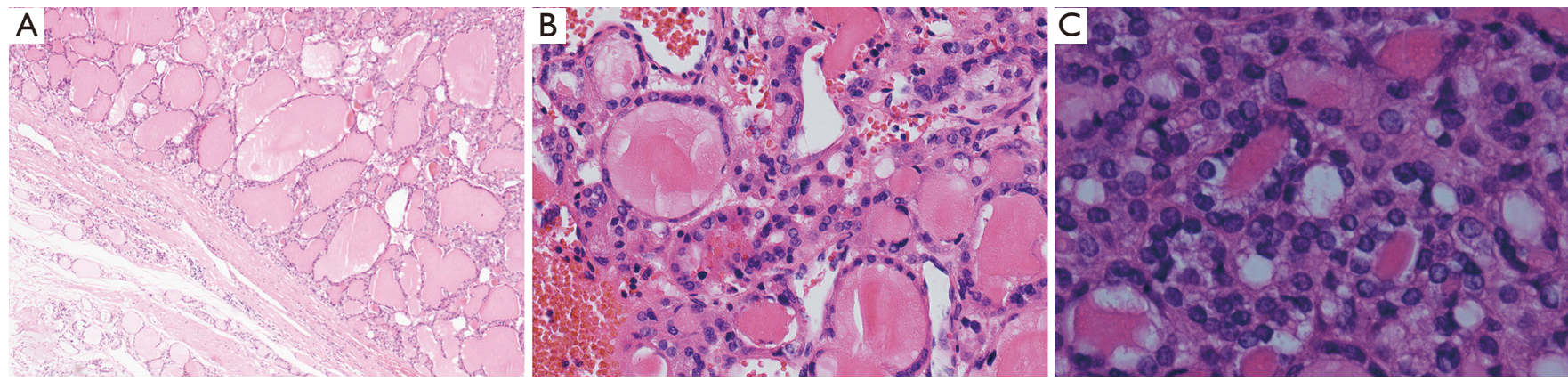

Figure 2 The histopathologic evaluation showed (A) a thyroid neoplasm with follicular growth pattern and without invasive signs (HE, $\times 40$ ); (B,C) cells lining the follicles demonstrated nuclear features of papillary thyroid carcinoma, while none papillae or psammoma bodies, necrosis and less than three mitoses per 10 high-power fields were observed (HE, B: $\times 200, \mathrm{C}: \times 400)$.

calcification, halo rim, extra-thyroidal-extension and color Doppler flow were documented during review. Size was defined as the maximum diameter of a NIFTP lesion, which was measured on longitudinal or transverse plane using internal calipers. The three US patterns of NIFTP were derived from these features: All solid lesions without suspicious features as calcifications, taller-than-wide shape and irregular margin were classified into type A. All partially cystic lesions were classified into type $\mathrm{B}$, regardless of other US features. In contrast, all hypoechoic solid lesions with suspected features mentioned above were classified into type C. All the NIFTP lesions were classified into the 3 major subtypes using pattern recognition. Moreover, the radiologists distributed all the NIFTP lesions to different categories based upon TIRADS (proposed by Kwak and ACR) and American Thyroid Association (ATA) guideline (14-16), and the disagreement would be solved by discussion and verdict of a specialists with over 20 years' experience. Kappa values for the inter-observer agreements of each US feature were calculated.

\section{Statistical analysis}

All statistical analyses were conducted using SPSS software (version 25.0, SPSS Chicago, IL, USA). The meta-analysis was performed using STATA 15.0 (Stata Corporation, College Station, TX, USA). The Forest Plots was plotted using package ggplot2 of R 3.6.3 (R foundation for Statistical Computing, www.R-project.org). The normality of variables was verified using a ShapiroWilk test. Biochemical variables were expressed as median and interquartile range, with Mann-Whitney test using for comparison. Other continuous variables were exhibited as mean $\pm \mathrm{SD}$ and compared using independent samples 
Student's $t$-test. Categorical variables were shown as counts and percentages and compared using chi-square statistics or the Fisher's exact test. Inter-observer agreements were assessed employing Cohen's Kappa statistics. Based on guidelines, kappa values were interpreted as follows: 0.00 0.20 , slight agreement; $0.21-0.40$, fair agreement; $0.41-0.60$, moderate agreement; $0.61-0.80$, strong agreement; and $0.81-1.0$, almost perfect agreement. A 2 -tailed $\mathrm{P}<0.05$ was viewed as threshold for statistically significant difference between two groups.

\section{Literature review and meta-analysis}

The PubMed and MEDLINE databases were searched for studies on sonographic features of NIFTP. We used search terms 'non-invasive follicular thyroid neoplasm with papillary-like nuclear features (NIFTP)' and 'ultra*', setting the search period between August 2016 and June 2020. Fifty-one studies were yielded and potentially eligible studies were selected by briefly reviewing of the titles and abstracts. The tables and outcomes in these studies were evaluated and those having requested data were included for analysis. Random effects meta-analysis was performed and the pooled estimates of proportions of each US feature were calculated.

\section{Results}

\section{Baseline characteristics}

Thirty patients with NIFTP lesions were eligible for inclusion in this study. Overall, $24(80.0 \%)$ patients were female, $6(20.0 \%)$ patients were male, and the mean age was $44.6 \pm 13.5$ years. Of these cases, $17(56.7 \%)$ cases were accompanied by papillary thyroid carcinoma, with 3 of follicular variant and 14 of classical variant. No significant difference in gender was observed between the macrogroup and micro-group $(\mathrm{P}=0.660)$. Six of thirty NIFTP specimens had gone through immunohistochemical analysis. The tumor cells were positive for CK19 in two cases, SDHB, CD56 and Cyclin D1 in one case. Ten patients (35.7\%) were found with comorbid Hashimoto's thyroiditis (Table 1$)$. Twenty patients $(66.7 \%)$ had received total thyroidectomy, 4 (13.3\%) patients treated with subtotal thyroidectomy and $6(20 \%)$ patients treated with lobectomy. Type A ( $n=19,63.3 \%)$ and type C ( $n=4,13.3 \%)$ US patterns were mostly presented in NIFTP nodules less than $1 \mathrm{~cm}$, while type $B(n=7,23.3 \%)$ US pattern was more frequent among NIFTP nodules larger than $1 \mathrm{~cm}$.

\section{Ultrasonography features}

Three patterns of US appearance were presented in our series (Figures 3-5). Type A: oval hypoechogenic, iso-or hyperechoic solid nodule with $(\mathrm{n}=7)$ or without halo $(\mathrm{n}=12)$; type B: partially cystic mass with halo $(\mathrm{n}=5)$ or without halo $(n=2)$; type C: hypoechogenic solid mass with suspected features like irregular margin $(\mathrm{n}=1)$ or microcalcifications $(\mathrm{n}=4)$. Amid all the NIFTP lesions, Hypoechogenic texture was found in 16 nodules, while Iso-or hyperechogenic texture was found in 12 nodules. The location of the tumor showed no preference between right or left lobes $(\mathrm{P}=0.264)$. Composition changed significantly between micro-group and macro-group, with partially cystic content appears more in macro-group and solid content distributes more in micro-group $(\mathrm{P}=0.024)$. Halo rim were highly prevalent in the macro-group $(\mathrm{P}=0.007)$. With regard to shape, no nodule exhibited nonparallel orientation or taller-than-wide shape in all the cases. Extrathyroidal extension (ETE) were negative for all the NIFTP nodules, either (Table 1). Of all nodules, none were beyond the classification system of the ATA, Kwak-TIRADS and ACR TIRADS guidelines. The detailed data were shown in the Figure 6, and notably, the classifications of all the 30 NIFTP lesions were consistent between ACR-TIRADS and Kwak-TIRADS. The interrater and original reports read agreement kappa value was showed in Table 2.

\section{Literature review and data synthesis}

Since the formally proposal of NIFTP in 2016, 12 publications on the ultrasonography of NIFTP were identified till June 2020 (11-13,17-25), one of which merely described the vascularity of NIFTP (19), and three of which generally reported the US features of NIFTP without requested details $(22,23,25)$. After evaluation, 6 studies consisted of required US features of NIFTP $(12,13,17,18,20,21)$. The pooled data of our cases combined with previous works were demonstrated in Table 3 and Figure 7. Overall, NIFTP nodules mainly presented solid composition, being either hypoechoic or iso-or hyperechoic, showing shape of wider-than-tall and circumscribed margin, less exhibiting calcifications, and with a significant fraction (50\%) sharing halo rim on US.

\section{Discussion}


Table 1 Sonographic, clinical and demographic details of NIFTP cases $(\mathrm{n}=30)$

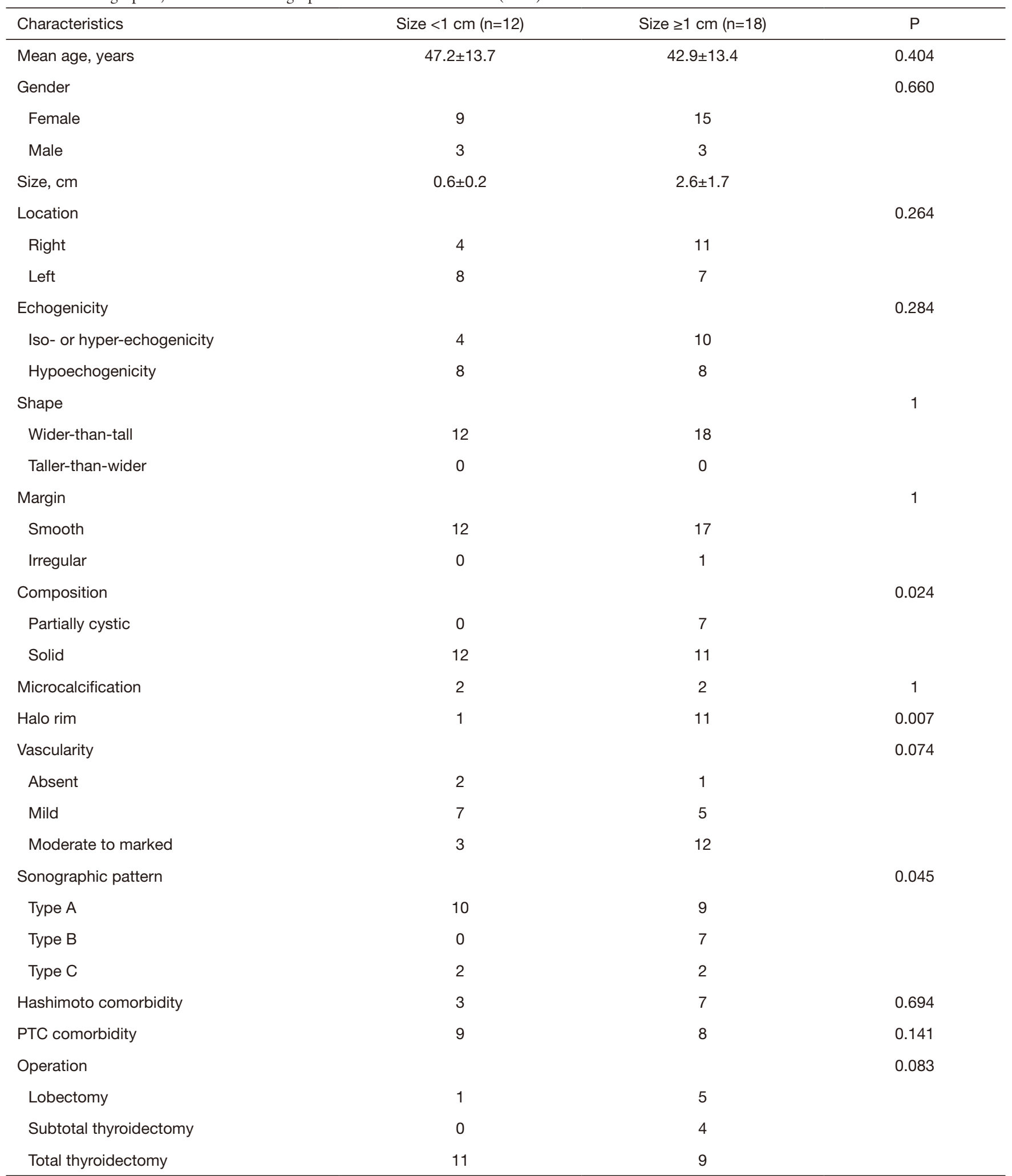

Immunohistochemical analysis: CK19 (2/6), SDHB (1/6), CD56 (1/6), Cyclin D1 (1/6). PTC, Papillary thyroid carcinoma; NIFTP, non-invasive follicular thyroid neoplasm with papillary-like nuclear features; $n$, number of cases. 

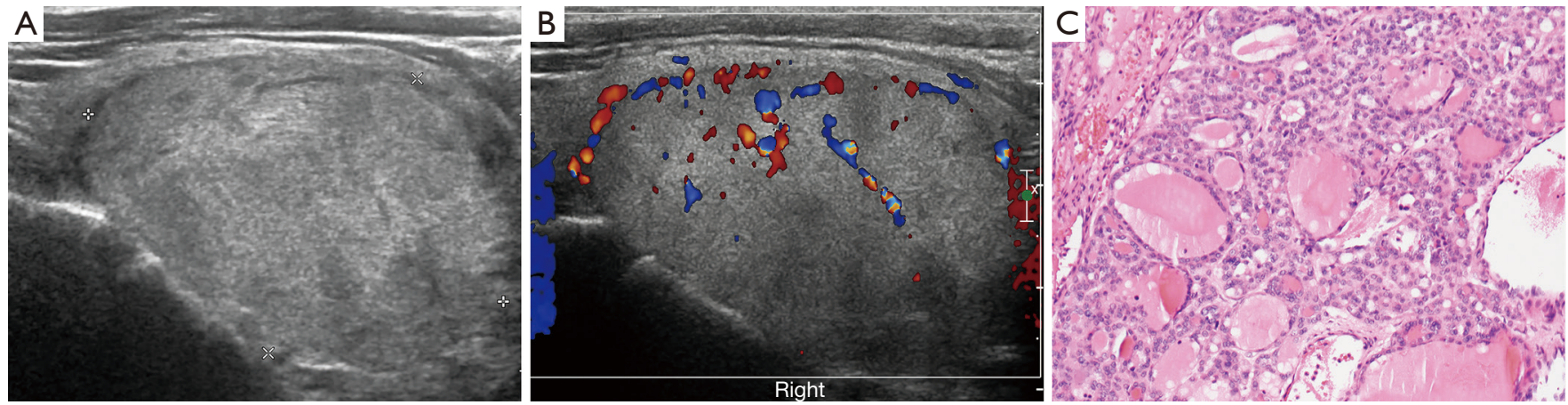

Figure 3 Longitudinal oriented B-mode imaging (A) and Color Doppler imaging (B) of type A NIFTP showing a solid isoechoic nodule surrounded by halo rim, without other suspicious features, accompanying with moderate vascularity. (C) HE stain, $\times 100$.
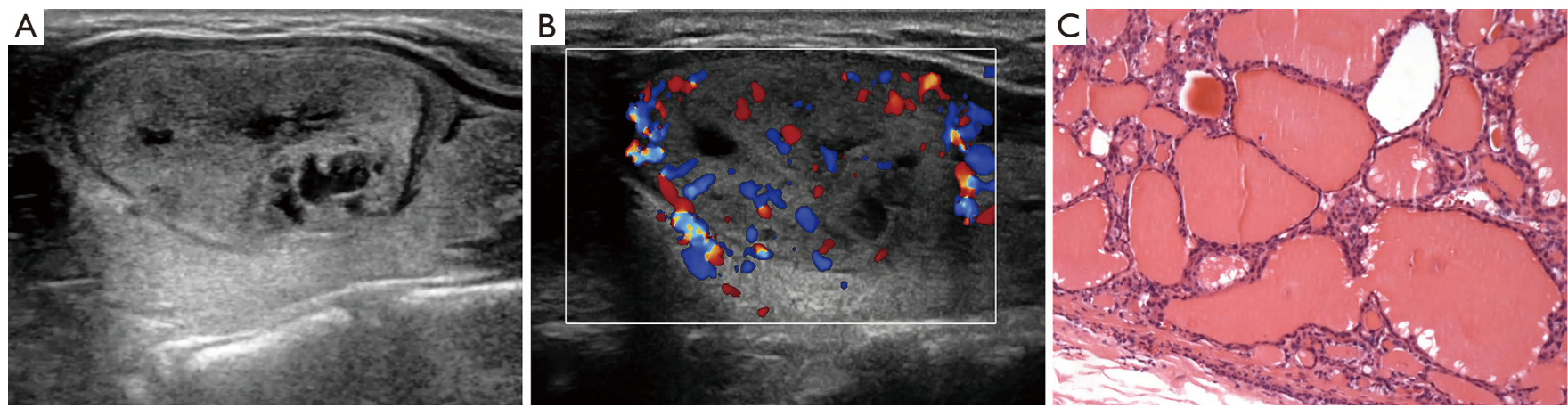

Figure 4 Longitudinal oriented B-mode imaging (A) and Color Doppler imaging (B) of type B NIFTP showing a partially cystic mass with halo rim and absence of other suspicious features, accompanying with marked vascularity. (C) HE stain, $\times 40$.
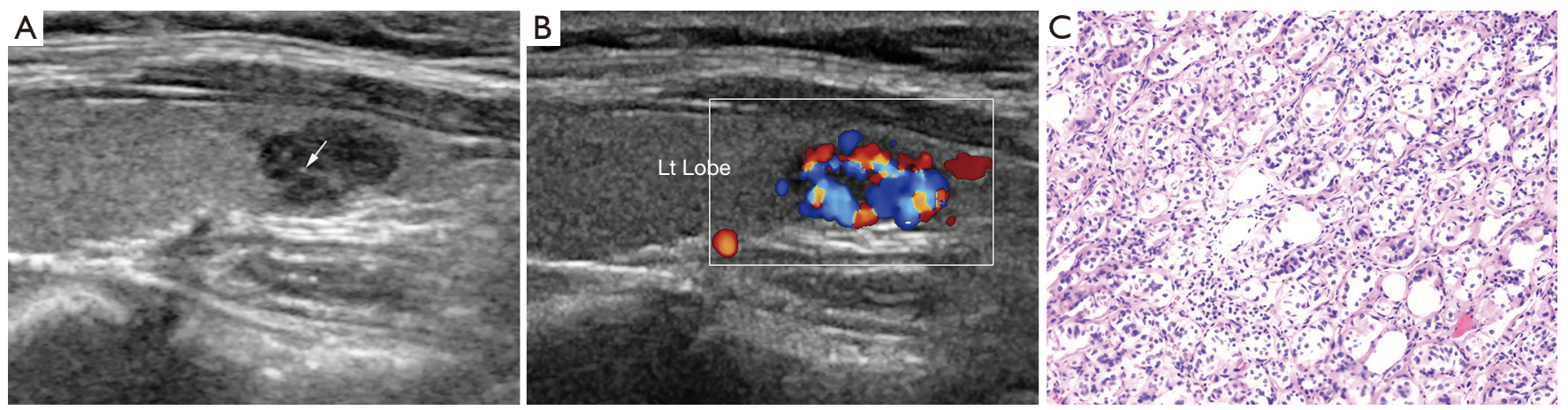

Figure 5 Longitudinal oriented B-mode imaging (A) and Color Doppler imaging (B) of type C NIFTP showing a solid hypoechoic nodule with microcalcifications (arrow), accompanying with marked vascularity. (C) HE stain, $\times 40$.

NIFTP was suggested to prevent overtreatment of thyroid carcinoma, but its current paradigm for treatment could not be overlooked in the heated debate. Lobectomy without adjuvant therapy is still the preferred choice of treatment for the entity. Ultrasound plays an essential role in the management of thyroid lesions. The ultrasound findings, to a great extent, determine the fate of a thyroid nodulefollow up, FNA or surgery. In this study, we found that NIFTP were mainly composed of three sonographic phenotypes. The solid composition and hypoechogenicity 

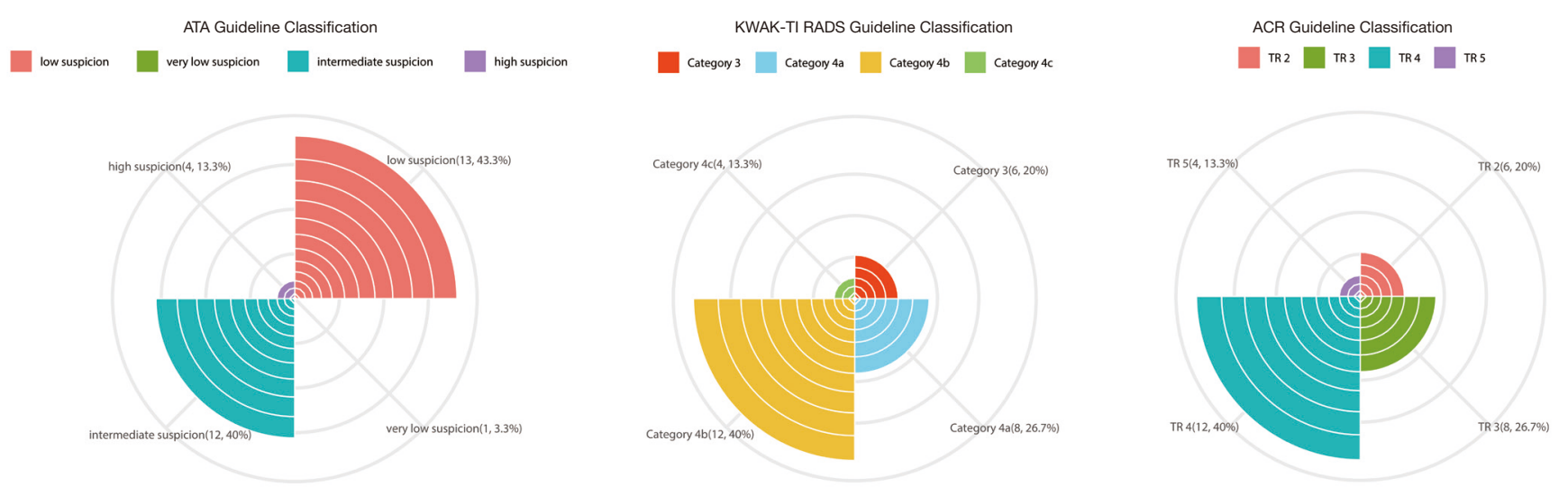

Figure 6 Classification results of each NIFTP lesion in this study using 2015 ATA guideline, Kwak-TIRADS and ACR TIRADS. NIFTP, non-invasive follicular thyroid neoplasm with papillary-like nuclear features; ATA, American Thyroid Association; Kwak-TIRADS, thyroid imaging, reporting and data system proposed by Kwak; ACR TIRADS, thyroid imaging, reporting and data system proposed by American College of Radiology.

Table 2 The concordance test between different raters and between original reports and final records

\begin{tabular}{|c|c|c|c|c|c|c|}
\hline Variability & Echogenicity & Shape & Margin & Composition & Calcification & Halo \\
\hline Records variability* & 0.664 & 0.651 & 0.366 & 0.627 & 0.672 & 0.585 \\
\hline
\end{tabular}

*, the variability between original records and the records re-evaluated and determined by two radiologists.

are the most common suspected features suggesting its potential malignancy.

The baseline characteristics of our NIFTP patients were consistent with previous reports of a preponderance of female and mean age of 40-50 years old. Immunohistochemical profiles of 6 NIFTP demonstrated positive staining in CK19 (2/6), SDHB (1/6), CD56 (1/6) and Cyclin D1 (1/6), which may add evidence to the differentiation between NIFTP and non-NIFTP lesions, as Cho et al. detected different expression in four markers of HBME-1, CK19, CD56, Galectin-3 between NIFTP and IFVPTC (26).

On the basis of size measured on ultrasonography, two groups of NIFTP were divided at the cutoff value of $1 \mathrm{~cm}$. We render such grouping meaningful and meeting the real-world demand because the less discussed $<1 \mathrm{~cm}$ NIFTP nodules may influence the incidence of NIFTP and management of some anxious patients who claim for FNA. Our analysis showed that NIFTP in micro-group mostly manifested as circumscribed solid oval nodule, which accorded with common perception of ultrasound appearance of NIFTP. However, we found that large
NIFTP lesions in macro-group exhibited more cystic change and halo rim compared with the micro-group counterparts. Thus, three US patterns were summarized from our data of NIFTP. Type-A, the most distinguished US pattern of NIFTP in previous reports, accounted for the largest proportion in our series as well. In line with our pooling data of a series of literature, this type of NIFTP consists of US feature appearing mostly frequent of its kind-solid composition (185/241, 76.7\%), parallel shape $(235 / 241,97.5 \%)$ and circumscribed margin $(226 / 241$, 93.7\%). Type-B was identified more frequently in macrogroup of our cases, which means that it may be associated with NIFTP in larger size. The pooling data showed that partially cystic change occupies $20 \%$ among all types of component, therefore, this US subset of NIFTP should not be ignored $(12,13,17,18,20,21)$. As for type-C, it contains NIFTP showing other suspected features, including irregular margin, nonparallel shape, and calcifications. Type-C US appearance often mimics that of papillary thyroid carcinoma at sonography for it had at least three suspected traits, but luckily, except for solid composition and hypoechogenicity, other suspected features are relatively 
Table 3 Pool estimates of ultrasound features of NIFTP in the literature from 2016 to 2020

\begin{tabular}{|c|c|c|c|c|c|c|c|c|}
\hline Ultrasound features & $\begin{array}{c}\text { Soo Yeon } \\
\text { Hahn }(n=34)\end{array}$ & $\begin{array}{l}\text { Sung-Hye } \\
\text { You }(n=45)\end{array}$ & $\begin{array}{l}\text { Hye Seung } \\
\text { Lee }(n=20)\end{array}$ & $\begin{array}{c}\text { Chanchal } \\
\text { Rana }(n=20)\end{array}$ & $\begin{array}{l}\text { Vincent } \\
\text { Larouche } \\
\quad(n=44)\end{array}$ & $\begin{array}{l}\text { Kwon } \\
(n=48)\end{array}$ & $\begin{array}{c}\text { Present } \\
\text { study } \\
(n=30)\end{array}$ & $\begin{array}{l}\text { Number (pooled } \\
\text { proportions) }\end{array}$ \\
\hline \multicolumn{9}{|l|}{ Composition } \\
\hline Solid & 29 & 29 & 18 & 10 & 41 & 35 & 23 & $185(78 \%)$ \\
\hline Cystic or predominantly cystic & 2 & 0 & 0 & 0 & 0 & 2 & 0 & $4(2 \%)$ \\
\hline \multicolumn{9}{|l|}{ Echogenicity } \\
\hline $\begin{array}{l}\text { Hypoechoic or marked } \\
\text { hypoechoic }\end{array}$ & 17 & 14 & 8 & 3 & 24 & 23 & 16 & $105(42 \%)$ \\
\hline Taller-than-wide (non-parallel) & 3 & 1 & 0 & 0 & 1 & 1 & 0 & $6(3 \%)$ \\
\hline Wider-than-tall (parallel) & 31 & 44 & 20 & 20 & 43 & 47 & 30 & $235(97 \%)$ \\
\hline \multicolumn{9}{|l|}{ Margin } \\
\hline Spiculated/microlobulated & 5 & 0 & 0 & 0 & 4 & 3 & 1 & $13(8 \%)$ \\
\hline Circumscribed & 29 & 45 & 20 & 20 & 40 & 43 & 29 & $226(92 \%)$ \\
\hline \multicolumn{9}{|l|}{ Calcification } \\
\hline Absent & 25 & 37 & 14 & 20 & 38 & 35 & 26 & 195 (80\%) \\
\hline
\end{tabular}

*, pooled proportions: pooled proportions of each US feature in NIFTP using random effects meta-analysis; ", these three together account for $20 \%$. NIFTP, non-invasive follicular thyroid neoplasm with papillary-like nuclear features; $n$, number of cases; NR, no report.

rare with percentage ranging from $2.5 \%$ to $7.5 \%$ according to our data aggregation $(12,13,17,18,20,21)$. The aggregated data also showed the echogenicity of NIFTP was unspecific with similar distribution between hypoecho/marked hypoecho and isoechoic/hyperecho (105/241, $43.5 \%$ vs. 136/241, 56.4\%). Yang et al. once explored the pathologic basis of ultrasound features of NIFTP and indicated that hypoechoic nodules may correlate to microfollicular/ solid components, while isoechoic/hyperechoic thyroid nodules may correlate to normofollicular/macrofollicular components. Therefore, it should bear in mind that the internal echogenicity varies in NIFTP and it should not disturb the recognition of the type-A and type-B US patterns in these lesions (27).

The prevalence of NIFTP varies in different regions and the underlying reasons remains unclear. Bychkov et al. demonstrated a very low proportion of NIFTP in nine series of Asian institutions: the mean rates of NIFTP was $1.5 \%$ (range $0-4.7 \%$ ) (28). In line with another four institutions in China (28-30), we observed the similar low rates of NIFTP $(0.30 \%)$ in our center and considered that rigid histopathological criteria of NIFTP could not account for the whole issue. Therefore, we assumed the confusions can be partly resolved from the perspective of sonography. To our knowledge of most Chinese institutes, patients with nodules showing in the macro-group usually 

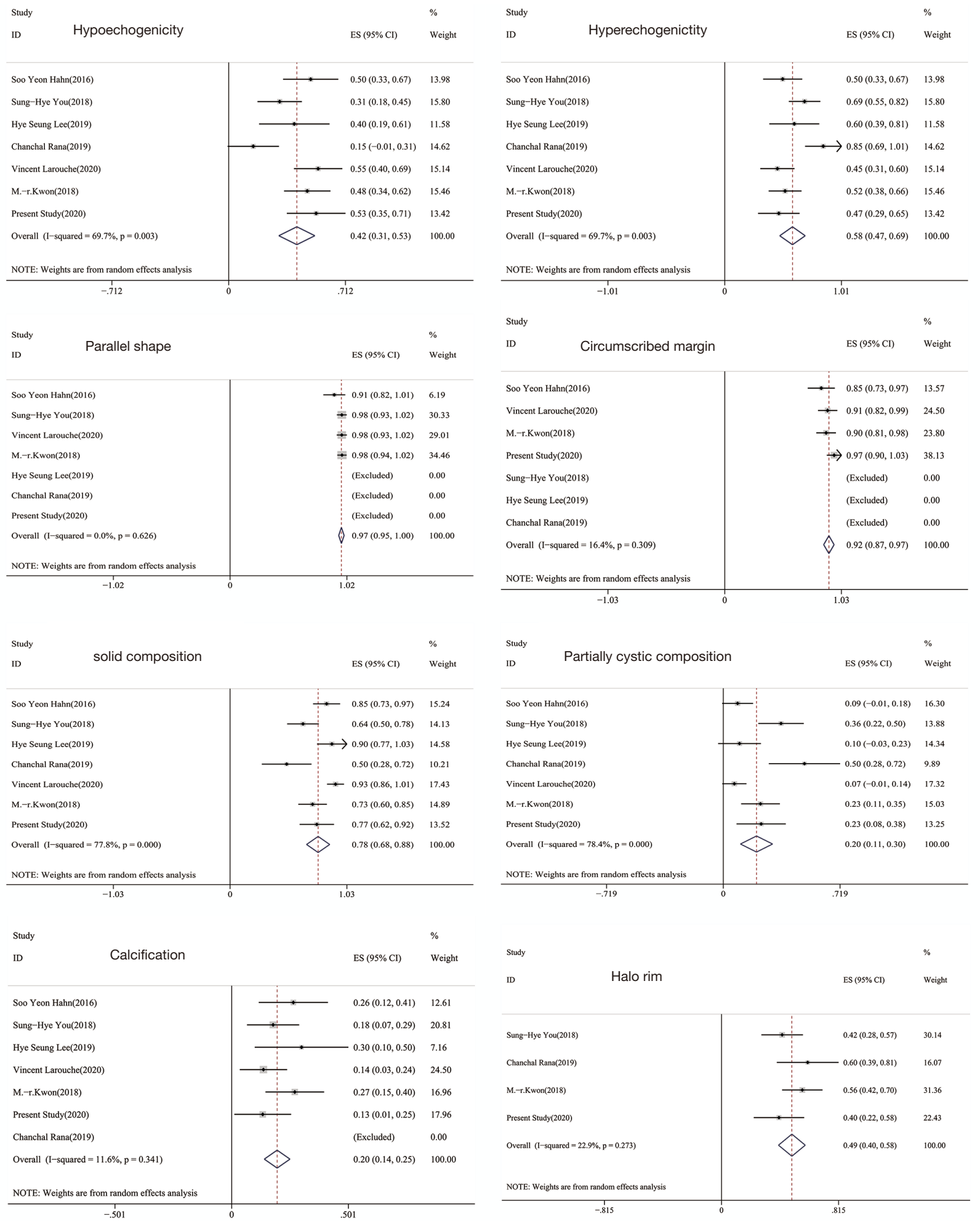

Figure 7 Forest plots of eight major ultrasound features related with NIFTP based on proportion meta-analysis. NIFTP, non-invasive follicular thyroid neoplasm with papillary-like nuclear features; ATA, American thyroid association; Kwak-TIRADS, Thyroid Imaging, Reporting and Data System proposed by Kwak; ACR TIRADS, Thyroid Imaging, Reporting and Data System proposed by American College of Radiology. 
get conclusion of probably benign lesions or possibly follicular lesions. Maybe out of the traditional conservative concepts of intervention in China, many patients opt for follow-up rather than FNA or surgery after consultation with specialists, let alone those with nodules $<1 \mathrm{~cm} \mathrm{(31).}$ Meanwhile, widely used sonographic classification systems in China including those of the American Thyroid Association, the American College of Radiology and Kwak hold different attitudes toward FNA of thyroid nodules and make the management of NIFTP deferred (32). Another observation worth mentioning is that more than half of the NIFTP in our series were accidentally identified during the total thyroidectomy of a thyroid carcinoma. That is, $50.0 \%(15 / 30)$ of NIFTP were not considered for operation initially, just resected as a whole in the wake of a papillary thyroid carcinoma. Canini et al. also found that $7.4 \%(5 / 68)$ cases with NIFTP were associated with classic PTC and 17.6\% (12/68) cases were diagnosed with NIFTP incidentally (33). These facts accounted for the rarity of NIFTP in our centre from another aspect. In this regard, we deemed that this newly defined entity may not exert as much influence as western countries in China. Zhu et al. also found NIFTP would not decrease risk of malignancy $(\mathrm{ROM})$ when "NIFTP $\neq \mathrm{Ca}$ " was in the analysis of 2,781 Chinese cases using The Bethesda System for Reporting Thyroid Cytology II (TBSRTC II) and supported our hypothesis $(29,34)$.

Our study has some limitations. Firstly, this study investigated NIFTP based on retrospective real-world observation. We could not fully control the diagnostic procedures with respect to ultrasound examination and pathology, which means the inter-observer variance may influence the final analysis. Secondly, the sample size in this study was small. Only a trend of difference in FNA rates was found among three guidelines. Further investigation of larger cohort is necessary to testify whether the difference is statistically significant or not. Thirdly, although literature review with data pooling was carried out to summarize US features of NIFTP, the three US subtypes of NIFTP need to be verified by every single case in different studies to avoid population bias. Lastly, we did not set internal nonNIFTP samples for comparison because the study mainly focused on summarizing ultrasound subtypes of NIFTP and guideline classifications of the entity rather than distinguishing NIFTP from other types of PTC. In the future, a large cohort study, drawing on new technologies like elastography and computer-aided diagnostic system, is expected to better evaluate the NIFTP and make it acknowledged in a didactic field $(35,36)$.

In conclusion, three US subtypes of NIFTP are summarized for imaging-pathology correlation analysis. Despite the widely accepted type-A US appearance of NIFTP, we also proposed the existence of type-B US presentation that accounts for not a little proportion of NIFTP, and the relatively rare series of type-C for reference. Ultrasonography evaluation also implies the underlying reason for low incidence of NIFTP in China: a large number of patients with thyroid lesions showing US features of NIFTP are probably under follow-up.

\section{Acknowledgments}

Funding: This study was supported by a grant from the Tibet Autonomous Region Science and Technology Project (XZ201901-GB-04).

\section{Footnote}

Reporting Checklist: The authors have completed the STROBE reporting checklist. Available at http://dx.doi. org/10.21037/gs-20-612

Data Sharing Statement: Available at http://dx.doi. org/10.21037/gs-20-612

Conflicts of Interest: All authors have completed the ICMJE uniform disclosure form (available at http://dx.doi. org/10.21037/gs-20-612). The authors have no conflicts of interest to declare.

Ethical Statement: The authors are accountable for all aspects of the work in ensuring that questions related to the accuracy or integrity of any part of the work are appropriately investigated and resolved. This study was approved by the Ethics Committee of Peking Union Medical College Hospital (No. S-K1304). Informed consent from enrolled patients was exempted by the IRB because of the retrospective nature of this study. The study was performed in accordance with the Declaration of Helsinki (as revised in 2013).

Open Access Statement: This is an Open Access article distributed in accordance with the Creative Commons Attribution-NonCommercial-NoDerivs 4.0 International License (CC BY-NC-ND 4.0), which permits the noncommercial replication and distribution of the article with 
the strict proviso that no changes or edits are made and the original work is properly cited (including links to both the formal publication through the relevant DOI and the license). See: https://creativecommons.org/licenses/by-nc-nd/4.0/.

\section{References}

1. Nikiforov YE, Seethala RR, Tallini G, et al. Nomenclature Revision for Encapsulated Follicular Variant of Papillary Thyroid Carcinoma: A Paradigm Shift to Reduce Overtreatment of Indolent Tumors. JAMA Oncol 2016;2:1023-9.

2. Geramizadeh B, Maleki Z. Non-invasive follicular thyroid neoplasm with papillary-like nuclearfeatures (NIFTP): a review and update. Endocrine 2019;64:433-40.

3. Rossi ED, Faquin WC, Baloch Z, et al. Noninvasive Follicular Thyroid Neoplasm with Papillary-Like Nuclear Features (NIFTP): Update and Diagnostic Considerations-a Review. Endocr Pathol 2019;30:155-62.

4. Nikiforov YE, Randolph GW, Ghossein RA, et al. Noninvasive follicular thyroid neoplasm with papillary-like nuclear features. In: Lloyd RV, Osamura RY, Klöppel $\mathrm{G}$, et al. (editors). WHO Classification of Tumours of Endocrine Organs. 4th ed. Lyon: IARC Press, 2017:78-80.

5. Parente DN, Kluijfhout WP, Bongers PJ, et al. Clinical Safety of Renaming Encapsulated Follicular Variant of Papillary Thyroid Carcinoma: Is NIFTP Truly Benign? World J Surg 2018;42:321-6.

6. Cho U, Mete O, Kim MH, et al. Molecular correlates and rate of lymph node metastasis of non-invasive follicular thyroid neoplasm with papillary-like nuclear features and invasive follicular variant papillary thyroid carcinoma: the impact of rigid criteria to distinguish non-invasive follicular thyroid neoplasm with papillary-like nuclear features. Mod Pathol 2017;30:810-25.

7. Bongiovanni M, Giovanella L, Romanelli F, et al. Cytological Diagnoses Associated with Noninvasive Follicular Thyroid Neoplasms with Papillary-Like Nuclear Features According to the Bethesda System for Reporting Thyroid Cytopathology: A Systematic Review and MetaAnalysis. Thyroid 2019;29:222-8.

8. Yan L, Sethi S, Park JW. Cytologic and clinical features of NIFTP: Can we diagnose based on preoperative fineneedle aspiration. Diagn Cytopathol 2019;47:1259-66.

9. Ruanpeng D, Cheungpasitporn W, Thongprayoon C, et al. Systematic Review and Meta-analysis of the Impact of Noninvasive Follicular Thyroid Neoplasm with Papillary-Like Nuclear Features (NIFTP) on Cytological
Diagnosis and Thyroid Cancer Prevalence. Endocr Pathol 2019;30:189-200.

10. Zhou H, Baloch ZW, Nayar R, et al. Noninvasive follicular thyroid neoplasm with papillary-like nuclear features (NIFTP): Implications for the risk of malignancy (ROM) in the Bethesda System for Reporting Thyroid Cytopathology (TBSRTC). Cancer Cytopathol 2018;126:20-6.

11. Rosario PW, Mourao GF, Nunes MB, et al. Noninvasive follicular thyroid neoplasm with papillary-like nuclear features. Endocr Relat Cancer 2016;23:893-7.

12. Hahn SY, Shin JH, Lim HK, et al. Preoperative differentiation between noninvasive follicular thyroid neoplasm with papillary-like nuclear features (NIFTP) and non-NIFTP. Clin Endocrinol (Oxf) 2017;86:444-50.

13. You SH, Lee KE, Yoo RE, et al. Prevention of total thyroidectomy in noninvasive follicular thyroid neoplasm with papillary-like nuclear features (NIFTP) based on combined interpretation of ultrasonographic and cytopathologic results. Clin Endocrinol (Oxf) 2018;88:114-22.

14. Haugen BR, Alexander EK, Bible KC, et al. 2015 American Thyroid Association Management Guidelines for Adult Patients with Thyroid Nodules and Differentiated Thyroid Cancer: The American Thyroid Association Guidelines Task Force on Thyroid Nodules and Differentiated Thyroid Cancer. Thyroid 2016;26:1-133.

15. Tessler FN, Middleton WD, Grant EG, et al. ACR Thyroid Imaging, Reporting and Data System (TI-RADS): White Paper of the ACR TI-RADS Committee. J Am Coll Radiol 2017;14:587-95.

16. Kwak JY, Han KH, Yoon JH, et al. Thyroid imaging reporting and data system for US features of nodules: a step in establishing better stratification of cancer risk. Radiology 2011;260:892-9.

17. Lee HS, Lee JW, Park JH, et al. Comprehensive analysis for diagnosis of preoperative non-invasive follicular thyroid neoplasm with papillary-like nuclear features. PLoS One 2019;14:e0218046.

18. Kwon MR, Shin JH, Hahn SY, et al. Histogram analysis of greyscale sonograms to differentiate between the subtypes of follicular variant of papillary thyroid cancer. Clin Radiol 2018;73:591.e1-7.

19. Rosario PW. Is Doppler ultrasonography of value for the differentiation between noninvasive follicular thyroid neoplasm with papillary-like nuclear features (NIFTP) and invasive encapsulated follicular variant of papillary thyroid 
carcinoma? Clin Endocrinol (Oxf) 2018;88:506-7.

20. Rana C, Manjunath S, Ramakant P, et al. Noninvasive follicular neoplasm with papillary like nuclear features: A comprehensive analysis with a diagnostic algorithm. Diagn Cytopathol 2020;48:330-41.

21. Larouche V, Pusztaszeri MP, Filimon S, et al. Preoperative prediction of non-invasive follicular thyroid neoplasm with papillary-like nuclear features: a Canadian single-Centre experience. J Otolaryngol Head Neck Surg 2020;49:1.

22. Rosario PW, da Silva AL, Nunes MB, et al. Risk of Malignancy in Thyroid Nodules Using the American College of Radiology Thyroid Imaging Reporting and Data System in the NIFTP Era. Horm Metab Res 2018;50:735-7.

23. Yang GCH, Fried KO, Scognamiglio T. Sonographic and cytologic differences of NIFTP from infiltrative or invasive encapsulated follicular variant of papillary thyroid carcinoma: A Review of 179 Cases. Diagn Cytopathol 2017;45:533-41.

24. Rosario PW. Ultrasonography and cytology as predictors of noninvasive follicular thyroid (NIFTP) neoplasm with papillary-like nuclear features: importance of the differential diagnosis with the invasive encapsulated follicular variant of papillary thyroid cancer. Clin Endocrinol (Oxf) 2017;87:635-6.

25. Boursier L, Clerc Urmes I, Garon J, et al. Ultrasound and cytological characteristics of non-invasive follicular thyroid neoplasm with papillary-like nuclear features compared to papillary carcinomas. Ann Endocrinol (Paris) 2020;81:28-33.

26. Cho H, Kim JY, Oh YL. Diagnostic value of HBME1, CK19, Galectin 3, and CD56 in the subtypes of follicular variant of papillary thyroid carcinoma. Pathol Int 2018;68:605-13.

27. Yang GCH, Fried KO. Pathologic basis of the sonographic differences between thyroid cancer and noninvasive follicular thyroid neoplasm with papillary-like nuclear

Cite this article as: Liu R, Gao L, Xia Y, Li X, Liao Q, Jiang Y, Zhang Q, Chen C, Zhang L, Liang Z, Jiang Y. Three ultrasound phenotypes of non-invasive follicular thyroid neoplasm with papillary-like nuclear features proposed for imaging-pathology analysis: single center experience. Gland Surg 2021;10(1):307-318. doi: 10.21037/gs-20-612 features. Ultrasonography (Seoul, Korea) 2018;37:157-63.

28. Bychkov A, Hirokawa M, Jung CK, et al. Low Rate of Noninvasive Follicular Thyroid Neoplasm with PapillaryLike Nuclear Features in Asian Practice. Thyroid 2017;27:983-4.

29. Zhu Y, Song Y, Xu G, et al. The Bethesda System for Reporting Thyroid Cytopathology (TBSRTC): A report of 2,781 cases in a Chinese population. Chinese Journal of Cancer Research 2020;32:140-8.

30. Ke J, Jianyong L, Ying L, et al. The use of The Bethesda System for Reporting Thyroid Cytopathology in a Chinese population: An analysis of 13351 specimens. Diagn Cytopathol 2019;47:876-80.

31. Kakudo K. How to handle borderline/precursor thyroid tumors in management of patients with thyroid nodules. Gland Surg 2018;7:S8-18.

32. Grani G, Lamartina L, Ascoli V, et al. Reducing the Number of Unnecessary Thyroid Biopsies While Improving Diagnostic Accuracy: Toward the "Right" TIRADS. J Clin Endocrinol Metab 2019;104:95-102.

33. Canini V, Leni D, Pincelli AI, et al. Clinical-pathological issues in thyroid pathology: study on the routine application of NIFTP diagnostic criteria. Sci Rep 2019;9:13179.

34. Cibas ES, Ali SZ. The 2017 Bethesda System for Reporting Thyroid Cytopathology. Thyroid 2017;27:1341-6.

35. Cantisani V, David E, Grazhdani H, et al. Prospective Evaluation of Semiquantitative Strain Ratio and Quantitative 2D Ultrasound Shear Wave Elastography (SWE) in Association with TIRADS Classification for Thyroid Nodule Characterization. Ultraschall Med 2019;40:495-503.

36. Fresilli D, Grani G, De Pascali ML, et al. Computeraided diagnostic system for thyroid nodule sonographic evaluation outperforms the specificity of less experienced examiners. J Ultrasound 2020;23:169-74. 